\title{
The Features of The Hyperbolic Slab Waveguide
}

\author{
E. I. Lyashko ${ }^{1}$ and A. I. Maimistov ${ }^{1,2 a}$ \\ ${ }^{1}$ Moscow Institute of Physics and Technology, 9 Institutskii lane, Dolgoprudny, Moscow region, 141700 Russia \\ ${ }^{2}$ National Research Nuclear University, Moscow Engineering Physics Institute, 31 Kashirskoe sh., Moscow, 115409 \\ Russia
}

\begin{abstract}
The dispersion relation determining guided TE and TM modes are found for the slab hyperbolic waveguide. The waveguide is consisting of an isotropic dielectric slab bounded by hyperbolic media. Some differences between the features of the waveguide under consideration and conventional ones are obtained. In particular, in the case of hyperbolic waveguide TM modes have two cutoff frequencies. As a result the number of modes is limited. Both TE and TM modes have nonzero cutoff frequencies, even though waveguide is symmetric one. . For the TE and TM modes the Poynting vector component along the wave's propagation axis could be equal to zero.
\end{abstract}

Keywords: metamaterials, hyperbolic media, waveguide modes

Many of the metameterials are anisotropic ones [1-3]. Recently the particular case of them referred as hyperbolic media are attracted the great attention [3-6]. A conventional anisotropic medium has the principal dielectric constants $\varepsilon_{o}$ and $\varepsilon_{e}$, which are positive. A hyperbolic medium exhibits the principal dielectric constants of different signs. A slab waveguide consisting of isotropic layer embedded in uniaxial hyperbolic medium is investigated in this research. The optic axis directed along normal to interface between the slab and its surroundings [7]. According this geometry TE wave is ordinary wave and TM wave is extraordinary one.

It was found that in the case of $\varepsilon_{o}>0, \varepsilon_{e}<0$ guided modes for TE waves are identical to modes for conventional waveguide. It is due to the TE waves are ordinary waves in this case. Whereas the TM wave radiation is not confined in this waveguide. In the case of hyperbolic medium featured by $\varepsilon_{o}<0, \varepsilon_{e}>0$ the propagation of the guided both TE and TM modes takes place. It is a reason to consider the hyperbolic medium with $\varepsilon_{o}<0, \varepsilon_{e}>0$ only.

Solution of the Maxwell equations with taking into account suitable boundary conditions results in an electromagnetic field distributions and dispersion relations for guided modes. In the case of symmetric waveguide the dispersion curves in terms of the normalized variables are represented in Fig.1. Here the following parameters are introduced. For the TE wave they are

$$
V=k_{0} h\left(n_{i}^{2}+n_{o}^{2}\right)^{1 / 2}, \quad b=\left(n_{o}^{2}+n_{e f}^{2}\right)\left(n_{o}^{2}+n_{i}^{2}\right)^{-1},
$$

\footnotetext{
${ }^{\text {a }}$ Corresponding author: aimaimistov@gmail.com
} 
and for TM wave

$$
V=k_{0} h\left(n_{i}^{2}-n_{e}^{2}\right)^{1 / 2}, \quad b=\left(n_{e}^{2}-n_{e f}^{2}\right)\left(n_{i}^{2}-n_{e}^{2}\right)^{-1} .
$$
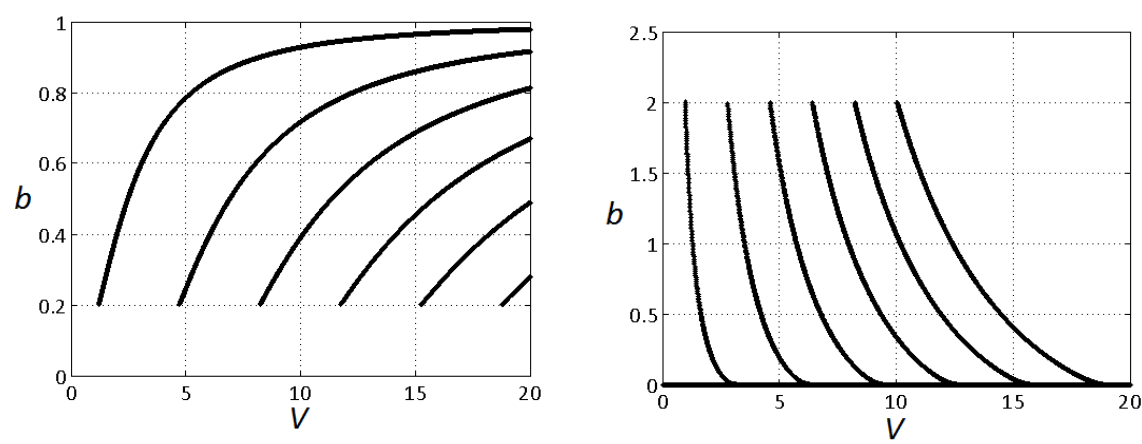

Figure 1. Dispersion relations for TE modes (left) and TM modes (right) in slab waveguide with hyperbolic substrate and clad.

These expressions contain the following definitions: $h$ is the isotropic layer thickness, $k_{0}=\omega / c$ is the wave number of vacuum, $n_{e f}$ is the effective waveguide index, $n_{i}$ is the refractive index of the isotropic layer, $n_{o}, n_{e}$, are the refractive indexes for ordinary and extraordinary waves, which are determined by the principal dielectric constants $\varepsilon_{o}$ and $\varepsilon_{e}$ of the substrate and clad of the slab waveguide under consideration.

Contrary to the conventional waveguide here all TE modes of symmetric waveguide has the non zero cutoff frequency. However, as usually the number of modes grows with increasing of the isotropic layer thickness.

The Fig. 1 shows that in the case of TM wave in hyperbolic waveguide the number of modes always is limited. With increasing of the isotropic layer thickness one of modes leaves waveguide, and another mode is appeared. For conventional waveguide the increasing of isotropic layer thickness results in the increasing of the number of guided modes. None of the mode is disappeared. The Fig.1 (right panel) shows that there are two cutoff frequencies for each TM mode propagating in a hyperbolic waveguide. One cutoff frequency at which the mode appears in waveguide with increasing the isotropic layer thickness, and the second cutoff frequency such that the mode is of no father confined in this waveguide. This second cutoff frequency does not exist in a conventional dielectric waveguide.

The definition of the longitudinal component of the wave vector in isotropic layer allows to write the effective waveguide index through angle $\vartheta$, which is effective incident angle on interface between isotropic layer and substrate: $n_{e f}^{2}=\varepsilon_{i} \sin ^{2} \vartheta$. Total reflection of the wave on this interface starts from the normal incident angle, i.e., $\vartheta=0$, and is finished at some critical incident angle. This is reason for existence of the two cutoff frequencies series.

The Poynting vector defines density of the radiation energy flux and direction of wave's energy propagation. It is instructive to consider an averaged projection of the Poynting vector along the $O Z$ axis. For TE wave it can be found from the equation

$$
\left\langle S_{z}\right\rangle=-\frac{c}{16 \pi}\left(E_{y}^{*} H_{x}+E_{y} H_{x}^{*}\right),
$$

and for TM wave it follows from the relation

$$
\left\langle S_{z}\right\rangle=-\frac{c}{16 \pi}\left(E_{x}^{*} H_{y}+E_{x} H_{y}^{*}\right)
$$


These relations can be rewritten with taking into account the spatial distributions for the TE wave and for the TM wave as

$$
\left\langle S_{z}\right\rangle_{T E}=\frac{c n_{e f}}{8 \pi \mu_{j}}\left|E_{y}\right|^{2}, \quad\left\langle S_{z}\right\rangle_{T M}=\frac{c n_{e f}}{8 \pi \varepsilon_{j}}\left|H_{y}\right|^{2},
$$

where $j=1, i, 3$ for $\mu_{j}$ and $j=e^{(1)}, i, e^{(3)}$ for $\varepsilon_{j}$ depending on the layer under consideration.

As was obtained in the previous two subsections, the effective index of refraction $n_{e f}$ for TE and TM guided modes in the hyperbolic waveguide can achieve null value. In these cases the averaged energy flux along the guided wave propagation axis $O Z$ will be zero. Thus, the effect of slowing light in the waveguide takes place in these cases.

Thus, the dispersion relations for the case of TE and TM waves are derived. It was shown that for the TM wave the number of guided modes is limited. Each of these modes have two cut-off frequencies. One of them corresponds to mode appearance, another corresponds to mode disappearance. There is region of parameters in which the only single mode exists in this waveguide. It is worth noting that this phenomenon is unavailable in the case of a conventional waveguide. Usually the number of modes increases with core thickness, and only single cut-off frequency exists. In the case of TM wave the effective index of refraction can be equal to zero, that leads to great phase velocity of the wave and zero Poynting vector component along the wave's propagation axis in the hyperbolic waveguide discussed above. Hence the light wave can be slowed down in this waveguide.

The research was supported by the Russian Science Foundation (Project No. 14-22-00098).

\section{References}

1. C.E. Kriegler, M.S. Rill, S. Linden, M. Wegener, IEEE Journal of Selected Topics in Quantum Electronics 16, No 2, P. 367-375 (2010)

2. V.P. Makarov, A.A. Rukhadze, JETP 103, No 3, 354-359 (2006)

3. M. A. Noginov, Yu. A. Barnakov et. al., Appl. Phys. Lett. 94, No 15. 151105 (2009)

4. A. Fang, Th. Koschny, C. M. Soukoulis, Phys.Rev. B 79, No 24, P. 245127 (2009)

5. V.P. Drachev, V.A. Podolskiy, A.V. Kildishev, Optics Express 21, No 12. P. 15048-15064 (2013)

6. Jingbo Sun, Jinwei Zeng, N. M. Litchinitser., Optics Express. 21, No 12. P. 14975-14981 (2013)

7. Satoshi Ishii; M.Y. Shalaginov, V.E. Babicheva, Al. Boltasseva, Al.V. Kildishev, Optics Letters 39, No 16. P. 4663-4666 (2014) 